$5-1-1992$

\title{
Dephasing Processes in Glasses with Strong Strain Interactions
}

Ulrich Zürcher

Cleveland State University, u.zurcher@csuohio.edu

R. Silbey

Massachusetts Institute o/Technology

Follow this and additional works at: https://engagedscholarship.csuohio.edu/sciphysics_facpub

Part of the Physics Commons

How does access to this work benefit you? Let us know!

Publisher's Statement

(C) 1992 American Institute of Physics.

\section{Repository Citation}

Zürcher, Ulrich and Silbey, R., "Dephasing Processes in Glasses with Strong Strain Interactions" (1992).

Physics Faculty Publications. 272.

https://engagedscholarship.csuohio.edu/sciphysics_facpub/272

This Article is brought to you for free and open access by the Physics Department at EngagedScholarship@CSU. It has been accepted for inclusion in Physics Faculty Publications by an authorized administrator of

EngagedScholarship@CSU. For more information, please contact library.es@csuohio.edu. 


\title{
Dephasing processes in glasses with strong strain interactions
}

\author{
U. Zürcher ${ }^{\text {a) }}$ and R. Silbey \\ Department of Chemistry, Massachusetts Institute of Technology, Cambridge, Massachusetts 02139
}

(Receivcd 2 August 1991; acceptcd 21 January 1992)

\begin{abstract}
Spectral diffusion decay is calculated for a glass modeled by two level systems which are strongly coupled to phonons. The spin-phonon interaction induces an effective spin-spin interaction which dominates the energy scale. We show that spectral diffusion is a property of macroscopic local fields which fluctuate on time scales that are much longer than the spinphonon relaxation time $T_{1}$. We assume for the spectral diffusion a Gaussian distribution and derive a self-consistent equation for its variance which is nonlocal in time. At high temperatures, the variance grows linearly with time while at low temperatures, we find strong deviations from simple diffusive decay. In a particular case, the growth of the variance is steplike. For very long times, we find an asymptotic sublinear behavior $w \propto t^{2 / 3}$. A heuristic argument shows that this law is determined by the form of the distribution.
\end{abstract}

\section{INTRODUCTION}

In solids, fast vibrations around local equilibrium configurations (phonons) lead to characteristic low temperatures properties like, e.g., the cubic dependence of the specific heat on temperature, $C_{v} \propto T^{3}$ for $T \rightarrow 0$. In amorphous glasses, on the other hand, the low temperature specific heat depends linearly on temperature, $C_{v} \propto T$ for $T \rightarrow 0$.' This and other thermal properties give evidence for additional degrees of freedom in glasses which are identified as changes in local configurations.

A widely used model assumes that a certain number of atoms (or group of atoms) has two low lying equilibrium positions: two level system (TLS) ${ }^{2.3}$ Strain fields in the glass cause the atoms to move from one equilibrium position to the other. In a mathematical description, the additional degrees of freedom are represented by spin-1 operators that are coupled to phonon creation and annihilation operators. Anomalous low temperature properties of glasses are explained in this model by assuming broad distributions for the spin level splittings and tunneling matrix elements.

Various dephasing experiments (optical hole burning, fluorescence line narrowing, etc.) probe dynamical processes in glasses from which additional insight into the nature of the TLS and their coupling to phonons can be inferred. ${ }^{4,5}$ Most theoretical treatments of dephasing processes ${ }^{4}$ use the concept of "spectral diffusion decay" as introduced by Klauder and Anderson. ${ }^{6}$

The exchange of virtual phonons induced by the spinphonon interaction gives rise to an effective TLS-TLS interaction which renormalizes the TLS energy splittings

$$
E^{k} \rightarrow E^{k}+H^{k} .
$$

Here, the local field is given by

$$
H^{k}=\gamma \sum_{l \neq k} \frac{1}{r_{k l}^{3}} o_{z}^{l}
$$

" Present address: Physics Department, Clarkson University, Potsdam, NY 13676.
In the standard TLS model, $H^{k}$ is assumed to be much smaller than $E^{k}$ so that the energy scale is dominated by the bare energy splittings. In this paper, we consider the opposite limit in which $H^{k} \gg E^{k}$.

The residual spin-phonon interaction leads to spin flips and thereby induces fluctuations in the local fields. In the standard terminology spins which have the same value $H_{0}$ of the local field at an initial time $t=0$ are called " $A$ spins" and are distinguished from all others (" $B$ spins"). In an inhomogeneously broadened line, $B$ spins outnumber $A$ spins. The spectral diffusion is governed by the conditional probability $p\left(H, t ; H_{0}, 0\right)$ that the local field has value $H$ at time $t$ if that value was $H_{0}$ at time $t=0$. For simplicity, we will label this probability as the spectral diffusion.

In discussion spin relaxation in solids, Klauder and Anderson $^{k}$ considered the weak coupling limit in which the phonon bath induces flips between unperturbed spin states with a rate $r=1 / T_{1}$ which they assume to be the same for all spins. Depending on the value of the minimal distance between neighboring spins, they found for the spectral diffusion either a Lorentzian or Gaussian form whose widths grow linearly in time

$$
w(t) \propto r t .
$$

That is, the spectral diffusion decays on the same time scale as the spins relax to thermal equilibrium.

However, experimental and theoretical studies have revealed that this is too simple a picture for glasses. In amorphous glasses, the spin population relaxation time $T_{1}$ is not constant but depends smoothly on intrinsic spin parameters. Black and Halperin calculated the spin relaxation in glasses using Fermi's golden rule. ${ }^{7}$ They showed that for short times, the width of the spectral diffusion depends strongly on temperature

$$
w(t) \propto T^{4} t .
$$

For phonon echo, saturation, and recovery, they find good qualitative and quantitative agreement between experimental results and their theoretical estimates.

Recently, Bai and Fayer pointed out an important dif- 
ference between optical dephasing experiments in simple solids and those in amorphous glasses. ${ }^{8}$ In simple solids, various optical dephasing experiments measure the same dephasing time $T_{2}$. The situation is entirely different in amorphous glasses where the observed dephasing time depends on the time scale associated with the experiment. In hole burning experiments, for example, the time scale is set by the waiting time between burning and reading the hole which can be as long as several hours, or even days. In their theoretical discussion of dephasing experiments, Bai and Fayer assume the spin population relaxation times lie in a certain time interval that is determined by the experiment. Therefore, different dephasing experiments measure different dephasing times, in general.

Still another time scale is set by intrinsic parameters of the system. A hole burning experiment in an amorphous host is modeled by a system consisting of the ground and excited state of the chromophore, TLSs describing the low lying excitations of the amorphous host, and a phonon bath. The linewidth of the chromophore is dominated by distant, weakly interacting TLS only if the (scaled) TLS-chromophore interaction is larger than the TLS relaxation rates (in units with $\hbar=1$ ). In this case, the expression for the linewidth does not explicitly depend on the chromophoreTLS interaction..$^{910}$ In the opposite limit, the chromophore linewidth will depend explicitly on the interaction of the chromophore with the nearby TLSs. In this paper we consider the former case for the linewidth of the chromophore.

While the existence of TLS degrees of freedom in glasses is well established, a detailed microscopic theory is still lacking; therefore, the TLS parameters are usually adjusted to give agreement with experiments. In Ref. 11, for example, the time evolution of a photochemical hole in an organic glass is reported to be much slower than the lifetime of the excited electronic state of the chromophore. The authors of Ref. 11 introduce TLS associated with photochemical proton transfer processes in addition to "normal" TLS which account for the observed thermodynamic anomalies of the glass. The relaxation rates of the fastest proton transfer processes are found to be orders of magnitude slower than the relaxation rates of the normal TLS. The distribution of relaxation rates of the additional TLS are assumed to be identical to the one describing the normal TLS. The observed optical spectral diffusion follows from the TLS spectral diffusion by averaging over the TLS relaxation rates. Because for the standard TLS model no clear justification is known, it is important to compare its predictions with those of alternative models.

We start with the observation that the definition of spectral diffusion contains the properties of the local field only. However, the $A$ spins all have different local environments. Therefore, the spectral diffusion is calculated by first considering the stochastic process $H(\tau), 0 \leqslant \tau \leqslant t$ at the site of an $A$ spin and then, in the second step, averaging over all different local environments of $A$ spins. Because for any $A$ spin, a single spin flip causes with equal probability a positive or negative change in the local field, the local field of the average $A$ spin changes much more slowly than the local field of an individual $A$ spin changes. That is, the local field of the average $A$ spin is a macroscopic variable which changes on much longer time scales than individual spins relax to thermal equilibrium.

In this paper, we calculate the spectral diffusion decay for a glass in which TLS are strongly coupled to strain fields. We show that strong spin-phonon coupling may explain slow macroscopic behavior in glasses whose microscopic degrees of freedom relax on much shorter time scales.

In independent studies, the possibility of strong spinphonon coupling has already been considered. Silbey and Kassner examined strain fields in glasses and found that the spin-phonon interaction is strong, not weak. ${ }^{12}$ They showed further that strong interaction gives a possible explanation for the broad distribution of internal TLS parameters. ${ }^{13} \mathrm{Yu}$ and Leggett went a step further, arguing that certain universal features of glasses can only be explained in a scenario in which the spin-phonon induced TLS-TLS interaction is essential. ${ }^{14}$ This scenario was used to explain the plateau in the thermal conductivity and the bump in the quantity $C_{v} / T^{3} \cdot{ }^{15}$ $\mathrm{Yu}$ and Leggett also suggest that collective modes are important for understanding other glass properties. In this paper, we pursue the traditional approach by using single spin quantities only.

The outline of the paper is as follows. In Sec. II, we sketch how the effective spin-spin interaction can be derived from a Hamiltonian that couples spins to a phonon bath. In Sec. III, we review the theory by Klauder and Anderson for spins which interact weakly with a phonon bath. Section IV contains the derivation of the self-consistent equation for the variance of the spectral diffusion. In Sec. V, we discuss the width for high and low temperatures and different distributions of the initial local fields. Finally, we summarize and discuss our main results in Sec. VI.

\section{MICROSCOPIC MODEL}

The Hamiltonian for a system of spins coupled to a phonon bath is given by, see, ${ }^{13}$

$$
\begin{aligned}
& \mathscr{H}=\mathscr{H}_{1}+\mathscr{H}_{2}+\mathscr{H}_{12}, \\
& \mathscr{H}_{1}=-\sum_{k} \frac{1}{2} E^{k} \sigma_{z}^{k}, \\
& \mathscr{H}_{2}=\sum_{q} \omega_{q} b_{q}^{+} b_{q}(\hbar=1), \\
& \mathscr{H}_{12}=\sum_{q, k}\left(\Delta_{q}^{k} \sigma_{x}^{k}+W_{q}^{k} \sigma_{x}^{k}\right)\left(b_{q}+b_{-q}\right) .
\end{aligned}
$$

Here, $\mathscr{H}_{1}$ is the Hamiltonian of the bare spins, $\mathscr{H}_{2}$ describes the phonon bath, and $\mathscr{H}_{12}$ is the spin-phonon interaction. For spins weakly interacting with the phonon bath, only the part proportional to $\sigma_{x}$, which induces spin flips, must be retained in the interaction $\mathscr{H}_{12}$.

The diagonal and off-diagonal elastic tensor, respectively, read is related to the deformation-potential tensor $D_{q}^{k}$ by, ${ }^{7,16}$

$$
\Delta_{q}^{k}=D_{q}^{k} \frac{\Delta^{k}}{E^{k}}, \quad W_{q}^{k}=D_{q}^{k} \frac{W^{k}}{E^{k}} .
$$

The energy splittings $E^{k}$ are given by 


$$
E^{k}=\sqrt{\left(\Delta^{k}\right)^{2}+\left(\dot{W}^{k}\right)^{2}} .
$$

The unitary transformation

$$
U=\exp \left[-\sum_{q, k} \frac{\Delta_{q}^{k}}{\omega_{q}} \sigma_{z}^{k}\left(b_{q}-b_{q}^{+}\right)\right],
$$

which introduces TLS dressed with virtual phonons, diagonalizes part of the spin-phonon interaction

$$
\begin{aligned}
\mathscr{H}= & -\sum_{k} \frac{1}{2} E^{k} \sigma_{z}^{k}+\sum_{q} \omega_{q} b_{q}^{+} b_{q} \\
& -\sum_{q, k, l} \frac{1}{\omega_{q}} \Delta_{q}^{k} \Delta_{-q}^{l} \sigma_{z}^{k} \sigma_{z}^{l} \\
& +\sum_{q, k} \frac{1}{4} W_{q}^{k}\left[\sigma_{+}^{k} \psi_{1}^{k}+\sigma_{-}^{k} \psi_{2}^{k}, b_{q}+b_{q}^{+}\right]+ \\
& -\sum_{q, k, l}\left(1-\delta_{k l}\right) \frac{1}{\omega_{q}} W_{q}^{k} \Delta_{-q}^{l}\left(\sigma_{+}^{k} \psi_{1}^{k}+\sigma_{-}^{k} \psi_{2}^{k}\right) \sigma_{z}^{l} .
\end{aligned}
$$

Here, $[,]_{+}$denotes the anticommutator,

$$
\psi_{n}^{k}=\exp \left[(-1)^{n} \sum_{q} \frac{2}{\omega_{q}} \Delta_{g}^{k}\left(b_{q}-b_{-q}^{+}\right)\right],
$$

and

$$
\sigma_{ \pm}^{k}=\sigma_{x}^{k} \pm i \sigma_{y}^{k}
$$

In Eqs. (2.8) and (2.9), we used the same notation for the transformed and original operators.

The fourth and fifth terms in Eq. (2.8) describe the residual spin-phonon interaction which induces flips of dressed spins. The third term is an interaction between dressed TLS.

A more general unitary transformation gives a $\sigma_{x}^{k} \sigma_{x}^{l}$ interaction as well. Such terms are, however, unimportant for our applications because they couple spins only which are in resonance with each other. ${ }^{16.7}$ The spin-spin interaction renormalizes the energy splitting between upper and lower spin states,

$$
E^{k} \rightarrow E^{k}+H^{k}
$$

where the local field $H^{k}$ is given by

$$
H^{k}=\gamma \sum_{l \neq k} \frac{1}{r_{k l}^{3}} \sigma_{z}^{l}
$$

\section{SPECTRAL DIFFUSION: WEAK COUPLING LIMIT}

It is instructive to summarize briefly the weak coupling theory by Klauder and Anderson. ${ }^{6}$ We start from the definition of the spectral diffusion,

$$
p\left(H, t ; H_{0}, 0\right)=\left\langle\delta\left(H-\gamma \sum_{i \neq k} \frac{\sigma_{z}^{l}}{r_{k l}^{3}}\right)\right\rangle,
$$

where the average $\langle\cdot\rangle$ is taken with respect to the conditional probability $\mathscr{P}\left(\left\{\sigma_{z}^{l}\right\}, t ;\left\{\sigma_{z}^{l}(0)\right\}, 0\right)$ that the spin variables have values $\sigma_{z}^{\prime}$ at time $t$ if their values were $\sigma_{z}^{\prime}(0)$ at time $t=0$. We insert the Fourier representation of the $\delta$ function and interchange averaging and integrating,
$p\left(H, t ; H_{0}, 0\right)=\frac{1}{2 \pi} \int_{-\infty}^{\infty} d y e^{i y H}\left\langle\exp \left(-i y \gamma \sum_{l \neq k} \frac{\sigma_{z}^{\prime}}{r_{k l}^{3}}\right)\right\rangle$

Because the spins are independent of each other, the average factorizes into a produce of single spin expectation values,

$p\left(H, t ; H_{0}, 0\right)=\frac{1}{2 \pi} \int_{-\infty}^{\infty} d y e^{i y H} \prod_{l \neq k}\left\langle\exp \left(-i y \gamma \frac{\sigma_{z}^{l}}{r_{k l}^{3}}\right)\right)$.

Assuming that the spin is initially in the state $\sigma_{z}^{l}(0)$ and jumps subsequently between its two levels with probability per unit time $r$, the single spin conditional probability is given by

$\mathscr{P}\left[\sigma_{z}^{l}, t ; \sigma_{z}^{l}(0), 0\right]=e^{-r t} \delta_{\sigma_{z}^{\prime} \sigma_{z}^{\prime}(0)}+\left(1-e^{-r t}\right) \mathscr{P}_{\text {eq }}$.

Here, $\mathscr{P}_{\text {eq }}$ is the equilibrium distribution

$$
\mathscr{P}_{\text {eq }}=\frac{1}{2}(|\uparrow\rangle\langle\uparrow|+| \downarrow\rangle\langle\downarrow|) \text {. }
$$

Inserting Eqs. (3.4) and (3.5) into Eq. (3.3), we find

$$
\begin{aligned}
p\left(H, t ; H_{0}, 0\right)= & \frac{1}{2 \pi} \int_{-\infty}^{\infty} d y e^{i j H} \prod_{l \neq k} \exp \left\{\left(-i y \gamma \frac{\sigma_{z}^{d}(0)}{r_{k l}^{3}}\right)\right. \\
& \left.\times\left[1-\left(1-e^{-r l}\right)\left(1-\cos \frac{y \gamma}{r_{k l}^{3}}\right)\right]\right\} .
\end{aligned}
$$

For short times, $t \rightarrow 0$, we find

$p\left(H, t ; H_{0}, 0\right)=\frac{1}{2 \pi} \int_{-\infty}^{\infty} d y \exp \left[i y\left(H-H_{0}\right)-r t K(y)\right]$,

where $K(y)$ is given by

$$
K(y)=\sum_{l \neq k}\left(1-\cos \frac{y \gamma}{r_{k l}^{3}}\right) .
$$

The function $K(y)$ is approximated by an integral,

$$
K(y)=4 \pi n \int_{r_{\min }}^{r_{\max }} d r r^{2}\left(1-\cos \frac{y \gamma}{r^{3}}\right),
$$

where $n$ is the density of $B$ spins. With no significant error, $r_{\max }$ is set equal to infinity. Depending on the value of $y$ we find,

$K(y)= \begin{cases}\left(2 \pi^{2} / 3\right) n \gamma|y|, & |y|>2 \pi r_{\min }^{3} / \gamma, \\ (2 \pi / 3) n(y \gamma)^{2} / r_{\min }^{3}, & y \rightarrow 0 .\end{cases}$

Thus, $K(y) \propto|y|$ except when $y$ is very small in which case $K(y) \propto y^{2}$.

Thus, for a small difference $H-H_{0}$, the conditional probability distribution is a Lorentzian whose width grows linearly with time

$$
w_{L}(t)=\frac{2 \pi^{2} \gamma}{3} n r t,
$$

while for $H-H_{0}$ large, the distribution is a Gaussian whose variance grows linearly with time

$$
w_{G}(t)=\frac{2 \pi \gamma^{2}}{3 r_{\min }^{3}} n r t
$$




\section{SPECTRAL DIFFUSION: STRONG COUPLING LIMIT}

We pointed out in Sec. I that spectral diffusion is a property of macroscopic fields, which change much more slowly than individual spins relax to thermal equilibrium. Thus, for calculating macroscopic properties, we replace the spin variables $\sigma_{z}^{k}(t)$ by their respective quantities averaged over a short time $\delta t$

$$
\sigma_{z}^{k}(t) \rightarrow \hat{\sigma}^{k}(t)=\frac{1}{\delta t} \int_{t}^{t+\delta t} d \tau \sigma_{z}^{k}(\tau), \quad \delta t \rightarrow 0 .
$$

The local field then reads, cf. Eq. (2.12),

$$
H^{h}(t)=\gamma \sum_{i \neq k} \frac{1}{r_{k l}^{3}} \hat{\sigma}^{l}(t)
$$

We note that the spin variables $\sigma_{z}^{k}(t)$ are discrete, $\sigma_{z}^{k}(t)= \pm 1$, while $\hat{\sigma}^{k}(t)$ are continuous

$$
-1<\hat{\sigma}^{k}(t) \leqslant+1 \text {. }
$$

Because the spins are coupled to a heat bath, the time averaged quantities $\hat{\sigma}^{k}(t)$ are sums of thermal expectation values and small deviations thereof,

$$
\hat{\sigma}^{k}(t)=\sigma_{\mathrm{eq}}^{k}\left[H^{k}(t)\right]+\delta \sigma^{k}(t) .
$$

In the notation, we explicitly indicated the dependence of the thermal expectation values on the instantaneous local fields at their respective sites,

$$
\sigma_{\text {eq }}^{k}\left(H^{k}\right)=\tanh \left(\frac{E^{k}+H^{k}}{2 T}\right)
$$

We assume for the small deviations that for different times $t \neq t^{\prime},\left\{\hat{\sigma}^{k}(t)\right\}_{k}$ and $\left\{\hat{\sigma}^{k}\left(t^{\prime}\right)\right\}_{k}$ are sets of independent random variables. That is, the random variables $\hat{\sigma}^{k}(t)$ are uncorrelated with respect to both different sites and different times.

Upon inserting Eq. (4.4) into Eq. (4.2), we obtain a decomposition of the local fields

$$
\begin{aligned}
H^{k}(t) & =\gamma \sum_{i \neq k} \frac{1}{r_{k l}^{3}} \sigma_{\mathrm{oq}}^{\prime}\left[H^{i}(t)\right]+\gamma \sum_{i \neq k} \frac{1}{r_{k l}^{3}} \delta \sigma^{l}(t), \\
& \equiv \bar{H}^{k}(t)+\delta H^{k}(t) .
\end{aligned}
$$

We identify the slowly varying parts $\bar{H}^{k}$ with macroscopic fields. The fluctuations $\delta H^{k}(t)$ determine the behavior of the local fields on short time scales which are, however, longer than the spin population relaxation time $T_{1}$.

Equations (4.6) and (4.7) show that, in principle, in the strong coupling limit, the local field at site $k$ depends on the local fields at all neighboring sites $l \neq k$ which, in turn, depend on the local field at site $k$. However, for simplicity, we neglect this correlation and treat the local fields as independent random variables.

\section{A. Short time behavior}

The fluctuations in the local fields $\delta H^{k}(t)$ are infinite sums of independent random variables, cf. Eq. (4.6). Under some additional weak conditions on the random variables $\delta \sigma^{k}(t)$, the law of large numbers shows that $\delta H^{k}(t)$ are Gaussian random variables. ${ }^{17}$ At different sites $k \neq k^{\prime}$, the contributions of a particular $\delta \sigma^{l}(t)$ have different weighing factors $1 / r_{k l}^{3}$ and $1 / r_{k^{\prime} l,}^{3}$, respectively. Thus, in lowest order of approximation, $\delta H^{k}(t)$ are independent Gaussian random variables. Further, at different times $t \neq t^{\prime}, \delta H^{k}(t)$ and $\delta H^{k}\left(t^{\prime}\right)$ are independent of each other, i.e., $\delta H^{k}(t)$ are Markov processes.

In summary, the short time fluctuations in the local fields are modeled by independent Gauss-Markov processes whose statistical properties are specified by their mean values

$$
\left\langle\delta H^{k}(t)\right\rangle_{s}=0,
$$

and correlation functions

$$
\begin{aligned}
& \left\langle\left[\delta H^{k}(t+\Delta t)-\delta H^{k}(t)\right]\left[\delta H^{l}(t+\Delta t)-\delta H^{l}(t)\right]\right\rangle_{s} \\
& \quad=\delta^{k l} D_{s}(T) \Delta t .
\end{aligned}
$$

Here, $\delta^{k l}$ is the Kronecker symbol ( $\delta^{k l}=1$ for $k=l$ and $\delta^{k l}=0$ for $k \neq l$ ) and $D_{s}$ is the short time diffusion constant which, in this theory, is an adjustable parameter dependent on the temperature of the phonon bath.

\section{B. Long time behavior}

The short time fluctuations in the local fields induce fluctuations in the thermal expectation values $\sigma_{\mathrm{eq}}^{k}\left[H^{k}(t)\right]$ which, in turn, determine the behavior of the macroscopic fields $\bar{H}^{k}(t)$, cf. Eq. (4.6). That is, the macroscopic fields are time dependent stochastic processes as well. Because the thermal expectation values $\sigma_{\text {eq }}^{k}\left[H^{k}(t)\right]$ depend on the macroscopic fields, the statistical properties of the macroscopic fields must be determined in a self-consistent way.

We consider the long time behavior of the local field at the site of an $A$ spin, and define the spectral diffusion by

$p\left(H, t ; H_{0}, 0\right)=\left\langle\left\langle\delta\left[H-\gamma \sum_{l \neq k} \frac{1}{r_{k l}^{3}} \sigma_{\text {eq }}^{l}\left[H^{\prime}(t)\right]\right]\right\rangle\right\rangle$.

Here, $\langle\langle\cdot\rangle\rangle$ denotes the average over both the short time fluctuations $\delta H^{l}(\tau), 0 \leqslant \tau \leqslant t$, and the long time fluctuations $\overline{I I}^{l}(\tau), 0 \leqslant \tau \leqslant t$, subject to the initial condition

$$
H_{0}=\gamma \sum_{l \neq k} \frac{1}{r_{k l}^{3}} \sigma_{\mathrm{eq}}^{l}\left[H^{l}(0)\right] \text {. }
$$

We insert the integral representation of the $\delta$ function and interchange integrating and averaging,

$$
\begin{aligned}
p\left(H, t ; H_{0}, 0\right)= & \frac{1}{2 \pi} \int_{-\infty}^{\infty} d y e^{i y H} \\
& \times\left\langle\left\langle\exp \left[-i y \gamma \sum_{l \neq k} \frac{1}{r_{k l}^{3}} \sigma_{\mathrm{eq}}^{l}\left[H^{\prime}(t)\right]\right]\right\rangle\right\rangle .
\end{aligned}
$$

Because we treat the local fields as independent random variables, we have

$$
\begin{aligned}
p\left(H, t ; H_{0}, 0\right)= & \frac{1}{2 \pi} \int_{-\infty}^{\infty} d y e^{i y H} \\
& \times \prod_{l \neq k}\left\langle\left\langle\exp \left[-i y \gamma \frac{1}{r_{k l}^{3}} \sigma_{\mathrm{eq}}^{l}\left[H^{t}(t)\right]\right]\right\rangle\right\rangle .
\end{aligned}
$$


The average $\left\langle\left\langle\exp \left[-i z \sigma_{\mathrm{eq}}[H(t)]\right]\right\rangle\right\rangle, z=y \gamma / r_{k l}^{3}$, is given by a cumulant expansion which we truncate after the second term, i.e.,

$$
\begin{aligned}
\left\langle\left\langle\exp \left[-i z \sigma_{\mathrm{eq}}[H(t)]\right]\right\rangle\right\rangle \simeq & \exp \left\{-i z \sigma_{\mathrm{eq}}[H(0)]\right. \\
& -\frac{z^{2}}{2}\left\langle\left\langle\left[\sigma_{\mathrm{eq}}[H(t)]\right.\right.\right. \\
& \left.\left.\left.\left.-\sigma_{\mathrm{eq}}[H(0)]\right]^{2}\right\rangle\right\rangle\right\} .
\end{aligned}
$$

We set $\left\langle\left\langle\sigma_{\text {eq }}[H(t)]\right\rangle\right\rangle=\sigma_{\text {eq }}[H(0)]$. To proceed we rewrite the second cumulant in an obvious way,

$$
\begin{aligned}
& \left\langle\left\langle\left[\sigma_{\mathrm{eq}}[H(t)]-\sigma_{\mathrm{eq}}[H(0)]\right]^{2}\right\rangle\right\rangle \\
& \quad=\left\langle\left\langle\left[\sum_{v=0}^{N} \sigma_{\mathrm{eq}}\left[H\left(\tau_{v+1}\right)\right]-\sigma_{\mathrm{eq}}\left[H\left(\tau_{v}\right)\right]\right]^{2}\right\rangle\right\rangle,
\end{aligned}
$$

where

$$
\begin{aligned}
& \tau_{0}=0, \\
& \tau_{N+1}=t .
\end{aligned}
$$

We choose equally spaced time steps,

$$
\tau_{v+1}-\tau_{v}=\Delta t=\frac{t}{N}
$$

so that $\Delta t \rightarrow 0$ as $N \rightarrow \infty$.

Because the macroscopic fields do not change during the short time interval $\left[\tau_{v}, \tau_{v+1}\right]$ we have, cf. Eq. (4.7),

$$
H\left(\tau_{v+1}\right)=\bar{H}\left(\tau_{v}\right)+\delta H\left(\tau_{v+1}\right)-\delta H\left(\tau_{v}\right) \text {. }
$$

Since $\left\langle\left[\delta H\left(\tau_{v+1}^{*}\right)-\delta H\left(\tau_{v}\right)\right]^{2}\right\rangle_{s}=D_{s} \Delta t \rightarrow 0$ as $\Delta t \rightarrow 0$, $\delta H\left(\tau_{v+1}\right)-\delta H\left(\tau_{v}\right)$ is typically a small quantity. Denoting $\sigma_{\mathrm{eq}}^{\prime}(H)=d \sigma_{\mathrm{eq}}(H) / d H$, we find to linear order in $\left[\delta H\left(\tau_{v+1}\right)-\delta H\left(\tau_{v}\right)\right]$,

$$
\begin{aligned}
\sigma_{\text {eq }} & {\left[H\left(\tau_{v+1}\right)\right]-\sigma_{\text {eq }}\left[H\left(\tau_{v}\right)\right] } \\
& =\sigma_{\text {eq }}^{\prime}\left[\bar{H}\left(\tau_{v}\right)\right]\left[\delta H\left(\tau_{v+1}\right)-\delta H\left(\tau_{v}\right)\right] .
\end{aligned}
$$

Inserting Eq. (4.20) into Eq. (4.15) gives

$$
\begin{aligned}
\left\langle\left\langle\left[\sigma_{\mathrm{eq}}[H(t)]-\sigma_{\mathrm{eq}}[H(0)]\right]^{2}\right\rangle\right\rangle \\
=\left\langle\left\langle\left[\sum_{v=0}^{N} \sigma_{\mathrm{eq}}^{\prime}\left[\bar{H}\left(\tau_{v}\right)\right]\left[\delta H\left(\tau_{v+1}\right)-\delta H\left(\tau_{v}\right)\right]\right]^{2}\right\rangle\right\rangle \\
=\sum_{v, \mu=0}^{N}\left\langle\sigma_{\mathrm{eq}}^{\prime}\left[\bar{H}\left(\tau_{v}\right)\right] \sigma_{\mathrm{eq}}^{\prime}\left[\bar{H}\left(\tau_{\mu}\right)\right]\right\rangle_{l} \\
\quad \times\left\langle\left[\delta H\left(\tau_{v+1}\right)-\delta H\left(\tau_{v}\right)\right]\right. \\
\left.\quad \times\left[\delta H\left(\tau_{\mu+1}\right)-\delta H\left(\tau_{\mu}\right)\right]\right\rangle_{s},
\end{aligned}
$$

where we have used the fact that the averages over long time fluctuations, $\langle\cdot\rangle_{l}$, and short time fluctuations, $\langle\cdot\rangle_{s}$, decompose. Using the statistical properties of the short time fluctuations, cf. Eqs. (4.8) and (4.9), we find

$$
\begin{aligned}
& \left\langle\left\langle\left[\sigma_{\mathrm{eq}}[H(t)]-\sigma_{\mathrm{eq}}[H(0)]\right]^{2}\right\rangle\right\rangle \\
& \quad=D_{s} \Delta t \sum_{v=0}^{N}\left\langle\left[\sigma_{\mathrm{eq}}^{\prime}\left[\bar{H}\left(\tau_{v}\right)\right]\right]^{2}\right\rangle_{1} .
\end{aligned}
$$

The average over the long time fluctuations is calculated by using the spectral diffusion

$$
\begin{aligned}
& \left\langle\left[\sigma_{\propto q}^{\prime}\left[\bar{H}\left(\tau_{v}\right)\right]\right]^{2}\right\rangle_{l} \\
& \quad=\int_{-\infty}^{\infty} d \bar{H} p\left(\bar{H}, \tau_{v} ; H_{0}, 0\right)\left[\sigma_{e q}^{\prime}(\bar{H})\right]^{2},
\end{aligned}
$$

where $H_{0}$ is the initial local field at that site. We insert Eq. (4.24) into Eq. (4.23) and let $N \rightarrow \infty$, and hence $\Delta t \rightarrow 0$, so that the sum goes over into an integral

$$
\begin{aligned}
& \left\langle\left\langle\left[\sigma_{e q}[H(t)]-\sigma_{\text {eq }}[H(0)]\right]^{2}\right\rangle\right\rangle \\
& \quad=D_{s} \int_{0}^{t} d \tau \int_{-\infty}^{\infty} d H p\left(H, \tau ; H_{0}, 0\right)\left[\sigma_{\text {eq }}^{\prime}(H)\right]^{2}
\end{aligned}
$$

We insert Eqs. (4.14) and (4.25) into Eq. (4.13),

$$
\begin{aligned}
p\left(H, t ; H_{0}, 0\right)= & \frac{1}{2 \pi} \int_{-\infty}^{\infty} d y e^{i y\left(H-H_{1}\right)} \\
& \times \prod_{l \neq k} \exp \left(-\frac{y^{2} \gamma^{2}}{2} \frac{1}{r_{k l}^{6}} D_{s} \int_{0}^{t} d \tau\right. \\
& \left.\times \int_{-\infty}^{\infty} d H p\left(H, \tau ; H_{0}^{l}, 0\right)\left[\left(\sigma_{\mathrm{eq}}^{\prime}\right)^{\prime}(H)\right]^{2}\right),
\end{aligned}
$$

where we have used

$$
H_{0}=\gamma \sum_{i \neq k} \frac{1}{r_{k l}^{3}} \sigma_{\mathrm{eq}}^{l}\left[H_{0}^{l}(0)\right]
$$

We average the expression on the right hand side (RHS) of Eq. (4.26) over the local environments. Because the spinspin interaction is assumed to dominate the energy scale, we set the bare level splitting equal to zero, $E^{k} \equiv 0$. Thus,

$$
\sigma_{\mathrm{eq}}^{k}\left(H^{k}\right)=\sigma_{\mathrm{eq}}\left(H^{k}\right)=\tanh \left(\frac{H^{k}}{2 T}\right) .
$$

At all spin sites, $\rho\left(H_{0}\right) d H_{0}$ is the probability that the initial local field has a value between $H_{0}$ and $I_{0}+d H_{0}$. Equation (4.26) is then

$$
\begin{aligned}
p\left(H, t ; H_{0}, 0\right)= & \frac{1}{2 \pi} \int_{-\infty}^{\infty} d y e^{i y\left(H-H_{11}\right)} \\
& \times \exp \left\{-\frac{y^{2} \gamma^{2}}{2} \cdot \sum_{l \neq k} \frac{1}{r_{k l}^{6}} \int_{-\infty}^{\infty} d H_{0} \rho\left(H_{0}\right)\right. \\
& \times \int_{0}^{t} d \tau \int_{-\infty}^{\infty} d H p\left(H, \tau ; H_{0}, 0\right) \\
& \left.\times\left[\sigma_{\text {cq }}^{\prime}(H)\right]^{2}\right\}
\end{aligned}
$$

Using

$$
\sum_{l \neq k} \frac{1}{r_{k l}^{6}} \rightarrow 4 \pi n \int_{r_{\min }}^{\infty} d r \frac{1}{r^{4}}=\frac{4 \pi}{3} \frac{n}{r_{\min }^{3}},
$$

where $r_{\min }$ is the shortest distance between different spins and $n$ is the density of $B$ spins, the sum over the spins is easily done, 


$$
\begin{aligned}
p\left(H, t ; H_{0}, 0\right)= & \frac{1}{2 \pi} \int_{-\infty}^{\infty} d y e^{i y\left(H-H_{0}\right)} \\
& \times \exp \left\{-\frac{4 \pi}{6} y^{2} \frac{\gamma^{2} D_{s} n}{r_{\min }^{3}} \int_{0}^{t} d \tau\right. \\
& \times \int_{-\infty}^{\infty} d H_{0} \rho\left(H_{0}\right) \int_{-\infty}^{\infty} d H p\left(H, \tau ; H_{0}, 0\right) \\
& \left.\times\left[\sigma_{\circ q}^{\prime}(H)\right]^{2}\right\} .
\end{aligned}
$$

We set

$$
\lambda(T)=\frac{8 \pi}{3} \frac{\gamma^{2} D_{s}(T) n}{r_{\min }^{3}}
$$

Performing the $y$ integration we find

$$
p\left(H, t ; H_{0}, 0\right)=\sqrt{\frac{1}{\pi w(t)}} \exp \left(-\frac{\left(H-H_{0}\right)^{2}}{w(t)}\right),
$$

where the variance of the Gaussian is given by

$$
\begin{aligned}
w(t)= & \lambda(T) \int_{0}^{t} d \tau \int_{-\infty}^{\infty} d H_{0} \rho\left(H_{0}\right) \\
& \times \int_{-\infty}^{\infty} d H p\left(H, \tau ; H_{0}, 0\right)\left[\sigma_{\text {eq }}^{\prime}(H)\right]^{2} .
\end{aligned}
$$

We comment on Eqs. (4.33) and (4.34). Most importantly, at time $t$, the variance of the spectral diffusion depends on the spectral diffusion at all previous times $0 \leqslant \tau \leqslant t$. This property stems from the interdependence of the equilibrium expectation values $\sigma_{\text {eq }}$ and local fields, cf. Eqs. (4.4)(4.7). The other points are of a more technical nature. The spectral diffusion is a Gaussian distribution in the difference $H-H_{0}$. This property is an approximation which is brought about by truncating the cumulant expansion after the sccond term, cf. Eq. (4.14), and setting $\ll \sigma_{\text {eq }}^{k}\left[H^{k}(t)\right] \gg$ $=\sigma_{\text {eq }}^{k}\left[H^{k}(0)\right]$. Further, the integrand in the $\tau$ integration is finite and positive definite. It follows that the variance has vanishing initial value, $w(0)=0$, increases monotonically with time, $w(t)>w\left(t^{\prime}\right)$ for $t>t^{\prime}$, and grows without bound. Hence, the spectral diffusion starts from an initial sharp distribution, $p\left(H, t=0 ; H_{0}, 0\right)=\delta\left(H-H_{0}\right)$, and decays monotonically for times $t>0$. Finally, the variance of the spectral diffusion depends on the distribution of the initial local fields.

In the next section, we discuss the variance $w(t)$ for particular choices of the initial distribution. We consider four different cases which indicate clearly that strong spinphonon coupling may lead to strong deviations from simple diffusive decay.

\section{VARIANCE OF THE SPECTRAL DIFFUSION}

Differentiating Eq. (4.34) with respect to time and using $\mathrm{Eq}$. (4.33) gives a first order differential equation

$$
\begin{aligned}
\frac{d w(t)}{d t}= & \lambda(T) \int_{-\infty}^{\infty} d H_{0} \rho\left(H_{0}\right) \frac{1}{\sqrt{\pi w(t)}} \\
& \times \int_{-\infty}^{\infty} d H \exp \left(-\frac{\left(H-H_{0}\right)^{2}}{w(t)}\right)\left[\sigma_{\mathrm{eq}}^{\prime}(H)\right]^{2}
\end{aligned}
$$

which together with the initial conditions, cf. the comments following Eq. (4.34),

$$
w(0)=0
$$

specifies the variance $w(t)$ of the spectral diffusion.

We choose for the distribution of the initial local fields $\rho\left(H_{0}\right)$ a form consisting of two identical Gaussians centered at $\widetilde{H}_{0}$ and $-\widetilde{H}_{0}$, respectively,

$$
\begin{aligned}
\rho\left(H_{0}\right)= & \frac{1}{2 \sqrt{\pi u}}\left\{\exp \left(-\frac{\left(H_{0}-\widetilde{H}_{0}\right)^{2}}{u}\right)\right. \\
& \left.+\exp \left(-\frac{\left(H_{0}+\widetilde{H}_{0}\right)^{2}}{u}\right)\right\} .
\end{aligned}
$$

We note that for $u \rightarrow 0, \rho\left(H_{0}\right)$ goes over into a sum of two $\delta$ functions,

$$
\rho\left(H_{0}\right)=\frac{1}{2}\left\{\delta\left(H_{0}-\widetilde{H}_{0}\right)+\delta\left(H_{0}+\widetilde{H}_{0}\right)\right\} .
$$

Inserting Eq. (5.3) into Eq. (5.1) gives

$$
\frac{d \tilde{w}(t)}{d t}=\frac{\lambda}{\sqrt{\pi \widetilde{w}(t)}} \int_{-\infty}^{\infty} d H \exp \left(-\frac{\left(H-\widetilde{H}_{0}\right)^{2}}{\tilde{w}(t)}\right)\left[\sigma_{\mathrm{eq}}^{\prime}(H)\right]^{2},
$$

where we have introduced

$$
\widetilde{w}(t)=w(t)+u \text {. }
$$

From Eqs. (5.6) and (5.2) we find the initial condition $\widetilde{w}(0)=u$.

In general, it is a formidable task to integrate Eq. (5.5) for $\widetilde{w}(t)$. It is only in the high temperature limit that an analytic expression for the variance can be found which is valid for all times. From Eq. (4.28) we have

$$
\sigma_{\text {eq }}^{\prime}(H) \simeq \frac{1}{2 T^{\prime}}, \quad T \rightarrow \infty .
$$

From Eqs. (5.5) and (5.7) we find $d \widetilde{w} / d t=\lambda / 4 T^{2}$. Hence, at high temperatures, the width of the spectral diffusion increases linearly with time as in the Klauder-Anderson model,

$$
w(t)=\frac{\lambda(T)}{4 T^{2}} t
$$

Here, we indicated explicitly that the parameter $\lambda$ is a function of temperature through its dependence on the short time diffusion constant $D_{s}$, cf. Eq. (4.32).

For finite temperatures, the behavior of $\sigma_{e q}^{\prime}(H)$ is nontrivial, cf. Eq. (4.28). However, $\sigma_{e q}^{\prime}(H)$ has simple asymptotic behavior for both small and large arguments. For $|H| \ll 2 T$, we find $\sigma_{\text {eq }}^{\prime}(H) \simeq 1 /(2 T)$ and for $|H| \gg 2 T$, we have $\sigma_{\text {eq }}^{\prime}(H) \simeq(2 / T) \exp (-H / T)$. An approximation for $\sigma_{\text {eq }}^{\prime}(H)$ is obtained by putting these two asymptotic forms together, 


$$
\left[\sigma_{e q}^{\prime}(H)\right]^{2} \simeq \begin{cases}1 / 4 T^{2}, & |H|<2 T \\ \left(4 / T^{2}\right) \exp (-2|H| / T) & |H|>2 T\end{cases}
$$

We note that the temperature of the phonon bath (in suitable units) defines the scale for the local fields. We insert Eq. (5.9) into Eq. (5.5)

$$
\begin{aligned}
& \frac{d \tilde{w}(t)}{d t} \\
& =\frac{\lambda(T)}{\sqrt{\pi \widetilde{w}(t)}} \frac{1}{4 T^{2}} \int_{-2 T}^{2 T} d H \exp \left(-\frac{\left(H-\widetilde{H}_{0}\right)^{2}}{\widetilde{w}(t)}\right) \\
& \quad+\frac{\lambda(T)}{\sqrt{\pi \widetilde{w}(t)}} \frac{4}{T^{2}}\left[\int_{2 T}^{\infty}+\int_{-\infty}^{-2 T}\right] d H \\
& \quad \times \exp \left(-\frac{2|H|}{T}\right) \exp \left(-\frac{\left(H-\widetilde{H}_{0}\right)^{2}}{\widetilde{w}(t)}\right) .
\end{aligned}
$$

In the following, we treat the cases for small and large width of the initial distribution separately. In both cases, the variance depends further on the average of the absolute values of the initial local fields.

\section{A. Narrow initial distribution}

We consider the case in which the second moment of the initial local fields is much smaller than the temperature,

$$
\sqrt{u} \ll 2 T \text {. }
$$

(a) Firstly, we set the average of the absolute values of the initial local fields much larger than the temperature,

$$
\widetilde{H}_{0} \gg 2 T \text {. }
$$

For these parameters, the distribution of the initial local fields consists of two Gaussians well inside the intervals $|H|>2 T$ in which $\sigma_{\text {eq }}^{\prime}(H)$ is exponentially small, cf. Eq. (5.9).

The temporal behavior of $\widetilde{w}(t)$ changes when the values of the local fields have finite probability to lie within the interval $|H|<2 T$. Thus, we define a crossover time $t_{1}$ by

$$
\sqrt{\widetilde{w}\left(t_{1}\right)}=\widetilde{H}_{0}-2 T .
$$

For times $t<t_{1}$, the integral on the RHS of Eq. (5.10) can be approximated to give

$$
\begin{aligned}
\frac{d \widetilde{w}(t)}{d t} \simeq & \frac{\lambda(T)}{\sqrt{\pi \widetilde{w}(t)}} \frac{4}{T^{2}} \int_{-\infty}^{\infty} d H \exp \left(-\frac{2 H}{T}\right) \\
& \times \exp \left(-\frac{\left(H-\widetilde{H}_{0}\right)^{2}}{\widetilde{w}(t)}\right) .
\end{aligned}
$$

It follows

$\frac{d \widetilde{w}(t)}{d t} \simeq \frac{4 \lambda(T)}{T^{2}} \exp \left(\frac{\widetilde{w}(t)}{T^{2}}-\frac{2 \widetilde{H}_{0}}{T}\right), \quad 0 \leqslant t \leqslant t_{1}$.

Simple integration gives for the variance $[w(t)$ $=\widetilde{w}(t)-u]$,

$$
w(t)=T^{2} \ln \left(\frac{1}{1-\left[4 \lambda(T) / T^{4}\right] \exp \left[-\left(2 \widetilde{H}_{0} / T-u / T^{2}\right)\right] t}\right), \quad 0 \leqslant t \leqslant t_{1} .
$$

From Eqs. (5.13) and (5.16), the crossover time $t_{1}$ is determined

$$
\begin{aligned}
t_{1}= & \frac{T^{4}}{4 \lambda(T)} \exp \left[\left(\frac{2 \widetilde{H}_{0}}{T}-\frac{u}{T^{2}}\right)\right] \\
& \times\left\{1-\exp \left[-\frac{\left[\left(\widetilde{H}_{0}-2 T\right)^{2}-u\right]}{T^{2}}\right]\right\} .
\end{aligned}
$$

For times much larger than the crossover time, $t \gg t_{1}$, the leading contribution to the integral on the RHS of Eq. (5.10) stems from the interval $|H|<2 T$. Setting $\exp \left[-\left(H-\widetilde{H}_{0}\right)^{2} / \widetilde{w}(t)\right] \simeq 1$ for $-2 T \leqslant H \leqslant 2 T$ we find

$$
\frac{d \widetilde{w}(t)}{d t} \simeq \frac{\lambda(T)}{\sqrt{\pi \widetilde{w}(t)}} \frac{1}{4 T^{2}} \int_{-2 T}^{2 T} d H=\frac{\lambda(T)}{\sqrt{\pi} T} \frac{1}{\sqrt{\widetilde{w}(t)}}, \quad t \gg t_{1} .
$$

Integration gives

$$
w(t) \simeq\left(\frac{3}{2 \sqrt{\pi}} \frac{\lambda(T)}{T}\right)^{2 / 3} t^{2 / 3}, \quad t \gg t_{1}
$$

where we used $w(t) \simeq \widetilde{w}(t)$ as $t \rightarrow \infty$. We note that the asymptotic long time behavior is independent of the parameters of the initial distribution. For this choice of the parameters $\widetilde{H}_{0}$ and $u$, the behavior of the variance $w(t)$ is sketched in Fig. 1.

We discuss briefly the temporal behavior of the variance $w(t)$. For very short times, the variance increases linearly in time

$$
w(t) \simeq \frac{4 \lambda(T)}{T^{2}} \exp \left[-\left(\frac{2 \widetilde{H}_{0}}{T}-\frac{u}{T^{2}}\right)\right] t, \quad t \rightarrow 0 .
$$

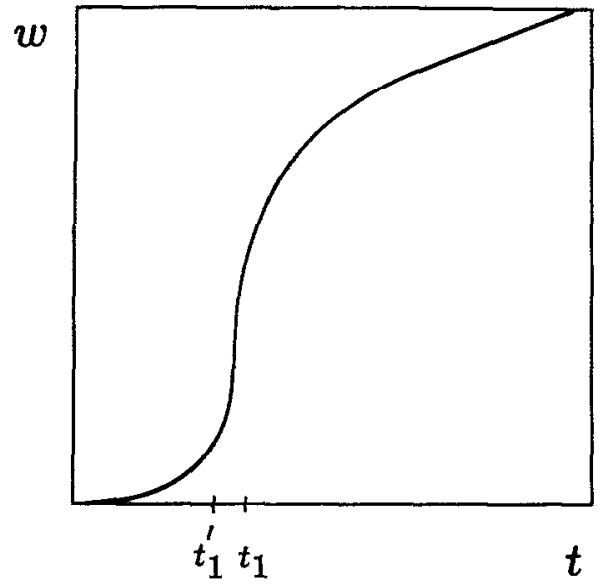

FIG. 1. The variance of the Gaussian distribution $w$ vs time $t$ (in arbitrary units) for the case in which the absolute values of the initial local fields are narrowly distributed around a large average value. 
We note that in Eq. (5.20) time is multiplied by a factor which is much smaller than that of the high temperature limit Eq. (5.8) continued to low temperatures. After this slow increase the variance increases much more rapidly, cf. Eq. (5.16). We define the time $t_{1}^{\prime}$ separating these two regimes by

$$
\frac{1}{\left(\widetilde{H}_{0}-2 T\right)^{2}} w^{\prime}\left(t_{i}^{\prime}\right)=1
$$

A calculation gives,

$$
\begin{aligned}
t_{i}^{\prime}= & \frac{T^{4}}{4 \lambda(T)} \exp \left[\left(\frac{2 \widetilde{H}_{0}}{T}-\frac{u}{T^{2}}\right)\right] \\
& \times\left\{1-\frac{4 \lambda(T)}{T^{4}} \frac{T^{2}}{\left(\widetilde{H}_{0}-2 T\right)^{2}}\right. \\
& \left.\times \exp \left[-\left(\frac{2 \widetilde{H}_{0}}{T}-\frac{u}{T^{2}}\right)\right]\right\}
\end{aligned}
$$

From Eqs. (5.17) and (5.22), we find

$$
\frac{t_{1}-t_{\mathrm{i}}^{\prime}}{t_{\mathrm{i}}^{\prime}} \simeq \frac{T^{2}}{\left(\widetilde{H}_{0}-2 T\right)^{2}} \frac{4 \lambda(T)}{T^{4}} \exp \left[-\left(\frac{2 \widetilde{H}_{0}}{T}-\frac{u}{T^{2}}\right)\right] \ll 1
$$

Using Eqs. (5.13) and (5.16), (5.22) we find further

$$
\frac{w\left(t_{1}^{\prime}\right)}{w\left(t_{1}\right)} \simeq \frac{2 T}{\widetilde{H}_{0}}<1
$$

Hence, in a steplike behavior, the variance grows rapidly in a short time interval. For times $t>t_{1}$, the variance grows with a slower rate, and approaches a sublinear behavior in the long time limit, cf. Eq. (5.19).

(b) Secondly, we maintain the assumption that the initial local fields are narrowly distributed, but now assume that the average of their absolute values is small. More specifically, we set

$$
\widetilde{H}_{0}+\sqrt{u}<2 T \text {. }
$$

The temporal behavior of $w(t)$ splits into two regimes. The crossover time $t_{2}$ is defined by

$$
\widetilde{H}_{0}+\sqrt{\widetilde{w}\left(t_{2}\right)}=2 T \text {. }
$$

For $t<t_{2}$, we find

$$
\begin{aligned}
\frac{d \widetilde{w}(t)}{d t} & =\frac{\lambda(T)}{\sqrt{\pi \widetilde{w}(t)}} \frac{1}{4 T^{2}} \int_{-\infty}^{\infty} d H \exp \left[-\frac{\left(H-\widetilde{H}_{0}\right)^{2}}{\widetilde{w}(t)}\right] \\
& =\frac{\lambda(T)}{4 T^{2}}=\text { const, } \quad t<t_{2} .
\end{aligned}
$$

Thus, we obtain,

$$
w(t)=\frac{\lambda(T)}{4 T^{2}} t, \quad t<t_{2}
$$

We note that the short time behavior has the same functional dependence as the high temperature limit Eq. (5.8). From Eqs. (5.26) and (5.28) the crossover time $t_{2}$ is given by

$$
t_{2}=\frac{4 T^{2}}{\lambda(T)}\left[\left(2 T-\widetilde{H}_{0}\right)^{2}-u\right] .
$$

For very long times, $t>t_{2}$, we find

$$
w(t) \simeq\left(\frac{3 \lambda(T)}{2 \sqrt{\pi} T}\right)^{2 / 3} t^{2 / 3}, \quad t \rightarrow \infty .
$$

This is the same long time behavior as in the case for large $\widetilde{H}_{0}$.

\section{B. Broad initial distribution}

We assume that the second moment of the initial local fields is much larger than the temperature

$$
\sqrt{u} \gg 2 T \text {. }
$$

One case of interest (and the only one considered here) is for a small value of $\widetilde{H}_{0}$.

$$
\text { - } \widetilde{H}_{0}<2 T \text {. }
$$

It follows that $\exp \left[-\left(H-\widetilde{H}_{0}\right)^{2} / u\right] \simeq 1$ for $|H|<2 T$. Thus, we find from Eq. (5.10),

$\frac{d \widetilde{w}(t)}{d t} \simeq \frac{\lambda(T)}{\sqrt{\pi \widetilde{w}(t)}} \frac{1}{4 T^{2}} \int_{-2 T}^{2 T} d H=\frac{\lambda(T)}{\sqrt{\pi} T} \frac{1}{\sqrt{\widetilde{w}(t)}}$.

We find for the variance

$$
w(t)=u\left(1+\frac{3 \lambda(T)}{2 \sqrt{\pi} T u^{3 / 2}} t\right)^{2 / 3}-u,
$$

where we used $w(t)=\widetilde{w}(t)-u$. We stress that this formula is valid for all times $t>0$. A Taylor expansion gives the short time behavior

$$
w(t) \simeq \frac{\lambda(T)}{\sqrt{\pi} T u^{1 / 2}} t, \quad t \rightarrow 0 .
$$

Since the radius of convergence for the Taylor expansion of $(1+z)^{\alpha}$ is $|z|=1$, the crossover time $t_{3}$ is given by

$$
t_{3}=\frac{2 \sqrt{\pi} T u^{3 / 2}}{3 \lambda(T)} \text {. }
$$

For long times, $t \gg t_{3}$, we find

$$
w(t) \simeq\left(\frac{3 \lambda(T)}{2 \sqrt{\pi} T}\right)^{2 / 3} t^{2 / 3}, \quad t \rightarrow \infty,
$$

which is the same behavior as that in the previous cases. In the next section, we elaborate more on the long time behavior.

\section{SUMMARY AND DISCUSSION}

We started from a model of a glass in which two level systems (spins) interact strongly with vibrational degrees of freedom (phonons). Strong spin-phonon interaction leads to an effective spin-spin interaction which dominates the energy scale. For simplicity, we then set the bare spin level splittings equal to zero. The spin-spin interaction brings about local fields which vary randomly from site to site and fluctuate in time due to the residual spin-phonon interaction. The spectral diffusion is defined as the conditional probability $p\left(H, t ; H_{0}, 0\right)$ that the local field has value $H$ at time $t$ if that value was $H_{0}$ at time $t=0$. We argued that this definition implicitly contains certain spatial averages. The spectral diffusion is, therefore, a property of macroscopic fields. This leads to a separation of time scales: individual spins relax to thermal equilibrium much faster than macroscopic fields 
change. Therefore, the spin variables are replaced by sums of equilibrium expectation values and small deviations thereof. In turn, this replacement induces a decomposition of the local fields into slowly and rapidly varying parts, respectively.

Although our approximate theory leaves many open questions about both the short and long time fluctuations, in this summary we focus on the long time fluctuations only. We showed that for each realization, at time $t$, the local field at site $k, H^{k}(t)$, depends on the histories $H^{l}(\tau), 0 \leqslant \tau \leqslant t$, at all neighboring sites $l \neq k$. These histories depend, in turn on the history of the local field at site $k, H^{k}(\tau)$ for $0 \leqslant \tau \leqslant t$. Hence, using probability distributions for local fields at single sites only, we neglected this possibly important correlation.

Our approximation theory rendered the spectral diffusion a Gaussian distribution in the difference $H-H_{0}$. For its variance we derived a self-consistent equation which is nonlocal in time and depends on the distribution of the initial local fields.

For high temperatures, on long time scales, the local fields spread diffusively $\left(\left\langle[H(t)-H(0)]^{2}\right\rangle \propto t\right)$ with a time constant which is in dependent of the initial distribution of the local fields and is reduced compared with its short time limit, $D_{l}=D_{s}(T) / 4 T^{2}$.

At low temperatures, the temporal behavior of the spectral diffusion decay depends on the distribution of the initial local fields. We found large deviations from simple diffusive behavior for the case in which the absolute values of the initial local fields are narrowly distributed around a large average value $\widetilde{H}_{0}$. During the initial time regime, which is long on macroscopic time scales, the spread of the local fields is well approximated by a simple diffusion process with a small time constant, $D_{l}=4 D_{s}(T) \exp \left(-\widetilde{H}_{0} / T\right) / T^{2}$. In the subsequent time regime, which is much shorter than the previous one, greatly enhanced fluctuations lead to a fast spread of the local fields. In the final time regime, the rate of change of the spread of the local fields decreases and the local fields approach an asymptotic subdiffusive behavior, $\left\langle[H(t)-H(0)]^{2}\right\rangle \propto t^{2 / 3}$. That is, we found a steplike behavior in which a large part of the spectral diffusion decay takes place in a short time. In the cases in which the absolute values of the initial local fields are narrowly distributed around a small average value and in which the distribution of the initial local fields is broad, the diffusive decay of the spectral diffusion for short times goes over smoothly into the same subdiffusive long time decay as in the above case.
The asymptotic long time behavior of the variance is determined by the center of the spectral diffusion, $p\left(H_{0}, t\right.$; $\left.H_{0}, 0\right)=\mathscr{P}[w(t)]$, and is, therefore, a consequence of the analytic form of the distribution. Indeed, the $t^{2 / 3}$ law is derived for a Gaussian distribution and becomes a $t^{1 / 2}$ law if a Lorentzian distribution is inserted into the RHS of Eq. (4.34). However, this equation is the correct expression for the variance of a Gaussian distribution only; its application to general distributions has to be more rigorously justified.

The observed logarithmic time dependence of spectral hole widths in some experiments can be explained by assuming a broad distribution of TLS relaxation rates in the Klauder-Anderson model, ${ }^{7}$ which comes from the distribution of the tunneling parameters. In this work, we have been concerned with the TLS relaxation rates due to TLS-TLS interactions. A complete theory would include both effects, and would consider correlations between the local fields at different spin sites as well. Such a treatment is, however, beyond the scope of this paper.

\section{ACKNOWLEDGMENTS}

U. Z. gratefully acknowledges partial financial support from the Swiss National Science Foundation which made his stay at M.I.T. possible. R. S. acknowledges the support from the National Science Foundation.

${ }^{1}$ R. C. Zeller and R. O. Pohl, Phys. Rev. B 4, 2029 (1971)

${ }^{2}$ P. W. Anderson, B. I. Halperin, and C. M. Varma, Philos. Mag. 25, 1 (1972).

${ }^{3}$ W. A. Phillips, J. Low Temp. Phys. 7, 351 (1972).

${ }^{4}$ Y. S. Bai, K. A. Littau, and M. D. Fayer, Chem. Phys. Lett. 162, 449 (1989); and references cited therein.

${ }^{5}$ P. J. van der Zaag, J. P. Galaup, and S. Völker, Chem. Phys. Lett. 166, 263 (1990)

"J. R. Klauder and P. W. Anderson, Phys. Rev, 125, 912 (1962).

${ }^{7}$ J. L. Black and B. I. Halperin, Phys. Rev. B 16, 2879 (1977).

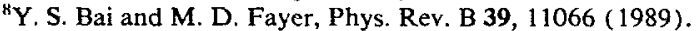

${ }^{9}$ L. W. Molenkamp and D. A. Wiersma, J. Chem. Phys. 83, 1 (1985).

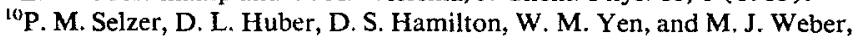
Phys. Rev. Lett. 36, 813 (1976).

"W. Breinl, J. Friedrich, and D. Haarer, J. Chem. Phys. 81, 3915 (1984).

${ }^{12}$ R. Silbey and K. Kassner, J. Lumin. 36, 283 (1987).

${ }^{13}$ K. Kassner and R. Silbey, J. Phys. Condensed Matter 1, 4599 (1989); K. Kassner, Z. Phys. B Condensed Matter 81, 245 (1990).

${ }^{14} \mathrm{C}$. C. Yu and A. J. Leggett, Comments Condensed Matter Phys. 14, 231 (1988).

tsC. C. Yu, Phys. Rev. Lett. 63, 1160 (1989).

inJ. Joffrin and A. Levelut, J. Phys. (Paris) 36, 811 (1975).

${ }^{17} \mathrm{C}$. W. Gardiner, Handbook of Stochastic Processes (Springer-Verlag, Berlin, 1983). 
The Journal of Chemical Physics is copyrighted by the American Institute of Physics (AIP). Redistribution of journal material is subject to the AIP online journal license and/or AIP copyright. For more information, see http:/ojps.aip.org/jcpo/jcpcr/jsp Copyright of Journal of Chemical Physics is the property of American Institute of Physics and its content may not be copied or emailed to multiple sites or posted to a listserv without the copyright holder's express written permission. However, users may print, download, or email articles for individual use. 
The Journal of Chemical Physics is copyrighted by the American Institute of Physics (AIP). Redistribution of journal material is subject to the AIP online journal license and/or AIP copyright. For more information, see http://ojps.aip.org/jcpo/jcper/jsp 